\title{
Etude et caractérisation des déchets ménagers de la ville de Mostaganem (Ouest-Algérie)
}

\section{GUERMOUD Noreddine et ADDOU Ahmed}

Laboratoire des sciences et techniques de l'environnement et de la valorisation (STEVA)

Faculté des Sciences et Sciences de l'ingénieur, BP 227, Université de Mostaganem, Algérie

\section{Auteur/s à qui la correspondance devrait être adressée : guermoud2700000@yahoo.fr}

\section{RESUME}

Notre étude porte sur la caractérisation des déchets ménagers de la ville de Mostaganem (Ouest Algérie) et ceci dans le but de proposer une ou plusieurs filières de valorisation. A cet effet, l'étude a été faite par quartier (secteur) et par saison. La collecte était assurée par le service de nettoiement de la Mairie (APC) de la ville de Mostaganem et acheminée au lieu de notre travail. Un tri manuel et minutieux est réalisé pour la caractérisation des déchets.

L'analyse des paramètres physico-chimiques (la densité, la perte au feu, la conductivité électrique, le pH moyen, le taux d'humidité de la matière fermentescible, le pouvoir calorifique inferieur $\mathrm{PCl}$, le carbone organique, l'azote) a été effectuée sur des échantillons obtenus en appliquant la méthode de quartage.

La production des déchets par habitant et par jour pour le citoyen de Mostaganem est de 0,82 kg.hab $\mathrm{b}^{-1 .-1}$.

La matière organique représente $65,5 \%$ des déchets, le papier et le carton 13\%, le plastique 7\%, le verre $4 \%$, les métaux $3,5 \%$, le textile $3 \%$, et les divers $4 \%$. Ces chiffres sont comparables à ceux des pays en voie de développement. L'étude en fonction des saisons a montré que la matière organique est passée à 80,3\% en été ce qui se justifie par la consommation des fruits et légumes alors que le papier chute à 8,3\%.

Les déchets ménagers de la ville de Mostaganem sont riches en matière organique (perte au feu de l'ordre de 62\% de la masse sèche) et azotée (1,4\%) et possèdent un taux d'humidité assez élevé (de l'ordre de $57 \%$ de la masse brute).

A partir de ces données, nous avons montré que $27,5 \%$ des déchets étaient potentiellement recyclables et que la filière de valorisation la plus appropriée pour les déchets de la ville de Mostaganem est le compostage. Cette étude préliminaire ouvre la voie à une prise de conscience par les autorités locales afin de mieux prendre en charge le problème des déchets et leur valorisation.

\section{ABSTRACT}

Our study focuses on the characterisazation of municipal solid waste in the city of Mostaganem (West Alegria) and this in order to introduce one of serval recovery processes (valorisation). For this purpose, the study was made by area (sector) and season. The collect was performed by the cleaning service of the city of Mostaganemand forwarded to the place of our work. Manual and meticulous sorting is performed for waste characterization.

Analysis of physico-chemical parameters (density, fire loss, electric conductivity, the average $\mathrm{pH}$, moisture level, lower calorific value, organic carbon and nitrogen) was performed on samples by applying the method of quartering.

Waste generation per inhabitant and per day for the citizens of Mostaganem is $0.82 \mathrm{~kg}$.inhab- I day- 1 .

The organic matter represents $65,5 \%$ from the waste, paper and cardboard $13 \%$, plastic $7 \%$, glass $4 \%$, metals 3,5\%, textile $3 \%$ and diverse $4 \%$. These figures are comparable to those of contries being development. The study according to the seasons showed that the organic matter went to $80,3 \%$ in summer which is justified by the consumption of vegetables and fruits while the paper fall to 8,3\%. Municipal solid waste of the city of Mostaganem are rich in organic matter (loss on ignition of about 62\% of the dry mass) and nitrogen (1.4\%) and have a relatively high moisture content (the around 57\% of the gross weight).

from these data, we showed that $27.5 \%$ were potentially recyclable waste, and die the most appropriate valuation for waste from the city of Mostaganem is composting. This preliminary study incite the local authority to take better care of the problem of waste and its valorization.

KEYWORDS : Municipal solid waste, Mostaganem, characterization, valorization 


\section{Introduction}

L'urbanisation et l'essor économique contribuent à l'amélioration des conditions de vie de l'individu, mais s'accompagnent d'une pollution de diverses natures nécessitant des mesures appropriées pour la neutraliser. D'ailleurs, les plus importantes des pollutions sont d'origine industrielle, commerciale, hospitalière, agricole ainsi que celles qui sont issues de la vie domestique appelés déchets ménagers ou encore ordures ménagères.

La pollution engendrée par ce genre de résidu est un problème très préoccupant et il est devenu un sujet d'actualité parce que les détritus délaissés n'importe où et n'importe comment constituent une véritable menace de notre environnement (MAYSTER, 1994). En effet, chaque jour, les ménages rejettent des tonnes de déchets, reflets de la consommation courante. II s'agit de nourriture ou de produits de la vie quotidienne, à cela s'ajoutent les déchets assimilables aux ordures ménagères. Cette quantité colossale d'ordures accumulées est à l'origine d'une multitude de nuisances dont les effets négatifs sont ressentis par la faune, la flore et l'homme (DOTROPPE, 1986). Si, dans les pays industrialisés, la gestion constitue déjà une activité économique très importante et convoitée, en Algérie par contre, la problématique des résidus n'est pas encore prise en charge. Les difficultés actuelles peuvent être attribuées à des facteurs multiformes d'ordre organisationnel, technique, institutionnel, éducationnel et a un manque d'information et de sensibilisation (MATE, 2003 ; GIROD, 1982). A notre connaissance très peu de données relatifs aux déchets ménagers existent et restent bloquer à l'échelle de la municipalité. La quantité de déchets ménagers produite en Algérie est estimée actuellement à $0,85 \mathrm{~kg}$.hab-I.j-I. Elle s'élève approximativement à 10,5 millions de tonnes par an. Cette quantité inclut les déchets ménagers ainsi que les déchets assimilables à des déchets ménagers et produits par l'activité municipale (balayures, déchets de marchés) et l'activité industrielle et commerciale (2,5 millions de tonnes générées par cette dernière activité) (GILLET, 1985 ; MATE et PROG$D E M, 2003)$. En matière de valorisation, notre pays enregistre un retard considérable (MATE, 2005). En effet, les déchets recyclables ne font en général pas l'objet d'une action organisée de tri, de récupération et de recyclage. La quantité de déchets recyclable est évaluée comme suit : 38500 tonnes lan de papier (50,6 \%) ; 130.000 tonnes/an de plastique $(17,1 \%) ; 100.000$ tonnes /an de métaux (13,2\%); 50.000 tonnes lan de verre $(6,5 \%) ; 95.000$ tonnes/an de matières diverses (12,5\%), soit un potentiel de matières recyclables de 760.000 tonnes estimé à 3,5 milliards de DA par an (MATE et PROGDEM, 2003).

Mostaganem, l'une des villes d'Algérie située au Nord-Ouest, souffre de ce genre de problème environnemental et rencontre d'énormes obstacles pour la gestion des déchets (MATE, 200I).

L'absence de données et le problème de gestion des déchets nous ont amenés à faire une étude sur la caractérisation et la détermination des paramètres physico-chimiques des déchets ménagers de la ville de Mostaganem afin de proposer une ou plusieurs filières de leur valorisation.

\section{Matériels et méthodes}

\section{I. Prélèvements et échantillonnage}

Pour déterminer la composition des ordures managers de la ville de Mostaganem, ainsi que leurs caractéristiques, Six secteurs (zone d'habitats) ont été choisis. Nous avons effectué des prélèvements directement à la source (ordures ménagères à l'état brut) durant la période allant de septembre 2009 à juillet 2010. L'étude a été faite en fonction des saisons et en tenant compte de :

\section{- La localisation}

- La densité de l'agglomération

- Les critères socio-économiques.

La collecte et l'acheminement des ordures ménagères à notre centre de tri étaient assurés par le service de nettoiement de la ville de Mostaganem. Six échantillons de $400 \mathrm{Kg}$ ont été collectés pour chaque secteur. Les véhicules de chargement des ordures ménagères ont été choisis en fonction de leur fréquence de rotation et de leur circuit de collecte et d'une manière aléatoire. Les secteurs sont représentés schématiquement par la figurel.

La quantité globale de déchet collecté de la ville de Mostaganem a été déterminée par pesage de 13 camions (Benne Tasseuse $12 \mathrm{~m}^{3} / 8 \mathrm{~m}^{3}$ ) à l'aide d'un pont bascule tout en tenant compte du nombre de rotation de chaque camion.

Dans le but de séparer par classe les déchets et de déterminer le poids de chaque catégorie : matière fermentescible, papier et carton, plastique et caoutchouc, textile, verre, métaux et divers (pierres, bois, etc.), nous avons effectué manuellement le tri. Pour la préparation des échantillons de laboratoire, les équipements nécessaires de sécurité ont été employés (masques, gants, lunettes) tandis que la réduction de la taille des matériaux était réalisée en utilisant des couteaux et des ciseaux de sorte que l'humidité contenue dans le déchet ne soit pas affectée. L'échantillon pour les analyses au laboratoire a été préparé en utilisant la méthode de quartage (triage, séchage, broyage) (GILLET, 1985).

\subsection{Analyses chimiques}

Le taux d'humidité ( $\mathrm{Hu}$ ) et la densité(d) ont été mesurés directement après déversement d'une quantité fraîche de $400 \mathrm{Kg}$ d'ordures ménagères au niveau du site d'étude (université de Mostaganem) en utilisant une balance technique de type Sauter SM 1600 et une étuve de séchage de type Memmert (GILLET, 1985 ; ZOES et al., 200 I). L'analyse de la matière sèche de l'échantillon de laboratoire nous a permis de déterminer les paramètres suivants :

\section{Etude et caractérisation des déchets ménagers de la ville de Mostaganem (Ouest-Algérie)}




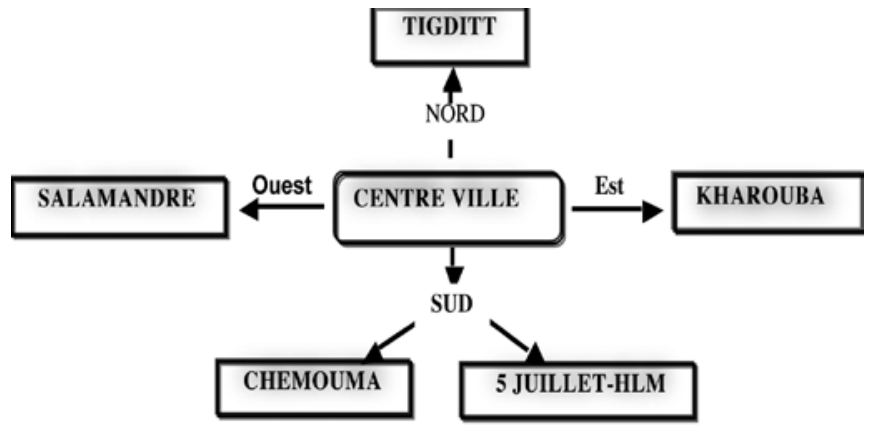

Figure I. Présentation des zones d'études.

La perte au feu (matière volatile) est déterminé après calcination à $550^{\circ} \mathrm{C}$ pendant $2 \mathrm{~h}$ dans un four de type Nabertherm (model L3/C6) (GILLET, 1985), le taux des cendres a été calculé en utilisant l'équation :\%Ce $=100-\% M v$, le carbone organique est déterminé par la méthode (SCHUMACHER, 2002 ; ANNE 1945), l'azote total est déterminé par la méthode de Kjeldhal modifiée (AUBERT, 1978), le pouvoir calorifique supérieur (PCS) a été mesuré expérimentalement à l'aide d'une bombe calorimétrique IKA C 4000 A (KHAN, 1991).

\section{Résultats et discussions}

\section{I. Composition moyenne des déchets ménagers de la ville de Mostaganem}

La quantité globale collectée par les opérateurs (publics et privés) intervenant dans la collecte des ordures ménagères et la quantité moyenne générée sont indiquées dans le tableau I (TAD, 2010).

La connaissance du ratio $\left(0,82 \mathrm{~kg}\right.$. $\left.\mathrm{Hab}^{-1 .-1}\right)$ ainsi que le nombre d'habitants 2010 nous a permis de calculer la quantité de déchet générée quotidiennement de la ville de Mostaganem et qui est différente de la quantité de déchets collectée.

La composition moyenne de déchets ménagers de la ville de Mostaganem a été obtenue en calculant la moyenne de celle de tous les secteurs étudiés. La matière organique est prédominante $(65,5 \%)$ par rapport aux autres composants de déchets (figure.2). Cela s'explique par le comportement nutritionnel des habitants qui favorisent la consommation des légumes et des fruits en raison de leur pouvoir d'achat qui correspond à un revenu plus ou moins moyen.

Les proportions du papier et du plastique sont moins importantes par rapport à la catégorie précédente, ils sont respectivement de l'ordre de 13\% et 7\%. La part des autres composants tels que les métaux, le verre et les divers constituent une fraction non négligeable résultant de l'activité des ménages

\subsection{Composition des déchets menagers en fonction des saisons}

Les opérations de tri manuel effectuées sur les lots d'ordures de chaque saison ont fourni les résultats qui sont regroupés dans le tableau 2. Le secteur de Chemouma a été choisi en raison de deux facteurs principaux, la forte densité de population et les différences de catégorie sociales.

L'examen de ce tableau révèle que la matière organique demeure prédominante par rapport aux autres catégories de déchets, elle atteint jusqu'à 80,3\% pendant la saison sèche avec un écart de 15,5\% par rapport à l'hiver. Ceci peut s'expliquer par le changement du régime alimentaire et aux habitudes du consommateur. Ils privilégient la consommation de fruits et légumes durant les saisons estivales, alors que pendant la saison hivernale, les citoyens favorisent les légumes secs.

\subsection{Composition des déchets ménagers en fontion des secteurs}

Les résultats d'analyse des échantillons collectés dans les différents secteurs sont regroupés dans le tableau 3.

La détermination du taux d'humidité des déchets ménagers est indispensable parce qu'elle permet d'apprécier et d'orienter la recherche vers un mode de traitement approprié. Cependant, l'examen des résultats montre que le taux d'humidité des ordures ménagères de la ville de Mostaganem demeure élevé, il varie de 52 à 64\% environ. On peut attribuer cette variation aux conditions climatiques et à la composition élémentaire de la matière putrescible qui pourrait affecter la teneur en eau des échantillons d'ordures ménagères.

Le rapport $\mathrm{C} / \mathrm{N}$ est un paramètre important qui permet d'apprécier l'aptitude des ordures au traitement par le compostage et renseigne sur la qualité du compost obtenu. Le rapport moyen $\mathrm{C} / \mathrm{N}$ est de l'ordre de 20. Cette valeur est dans la plage [20-30] considérée comme optimale (BAYARD, 2007 ; DOETSCH et al., 2002 ; GIROD, 1982 ; HAUG, 1980) pour l'obtention d'un compost de qualité. Le pouvoir calorifique supérieur (PCS) est un paramètre important pour apprécier l'aptitude du déchet à être incinéré. Les résultats d'analyse obtenus indiquent que ce paramètre reste faible ( $430 \mathrm{Kcal} / \mathrm{Kg}$ ) par rapport à celui préconisé pour l'incinération des déchets ( $1700 \mathrm{Kcal} / \mathrm{Kg})(\mathrm{KHAN}, 1991)$.

\subsection{Teneur en matériaux potentiellement recyclables}

Les proportions des matières recyclables (papier-carton, plastique, verre, métaux) contenues dans les ordures ménagères de la ville de Mostaganem ont été déterminées à partir des quantités générées par secteur et durant les quatre saisons. Ils représentent 27,5\% des déchets ménagers. Les résultats obtenus sont présentés sous forme d'histogrammes comme le montre la figure 3.

Ce taux représente presque le 1/3 de la masse globale des déchets ménagers de la ville de Mostaganem. Une étude comparative a été faite avec certaines villes algériennes et autres du monde (tableau 4), elle montre que le taux moyen de déchets de l'Algérie est de l'ordre de 27,2\%. Cette valeur est moins importante par rapport à celle de la Jordanie et la Turquie. 
Tableau I. Le tonnage moyen de déchets générés et collectés.

Commune de Quantité générée

Quantité collectée

Population

Ratio

Mostaganem

par jour (T) par jour (T) 2010 Kg.hab-I.j-I
133,2
128,6

162418

0,82

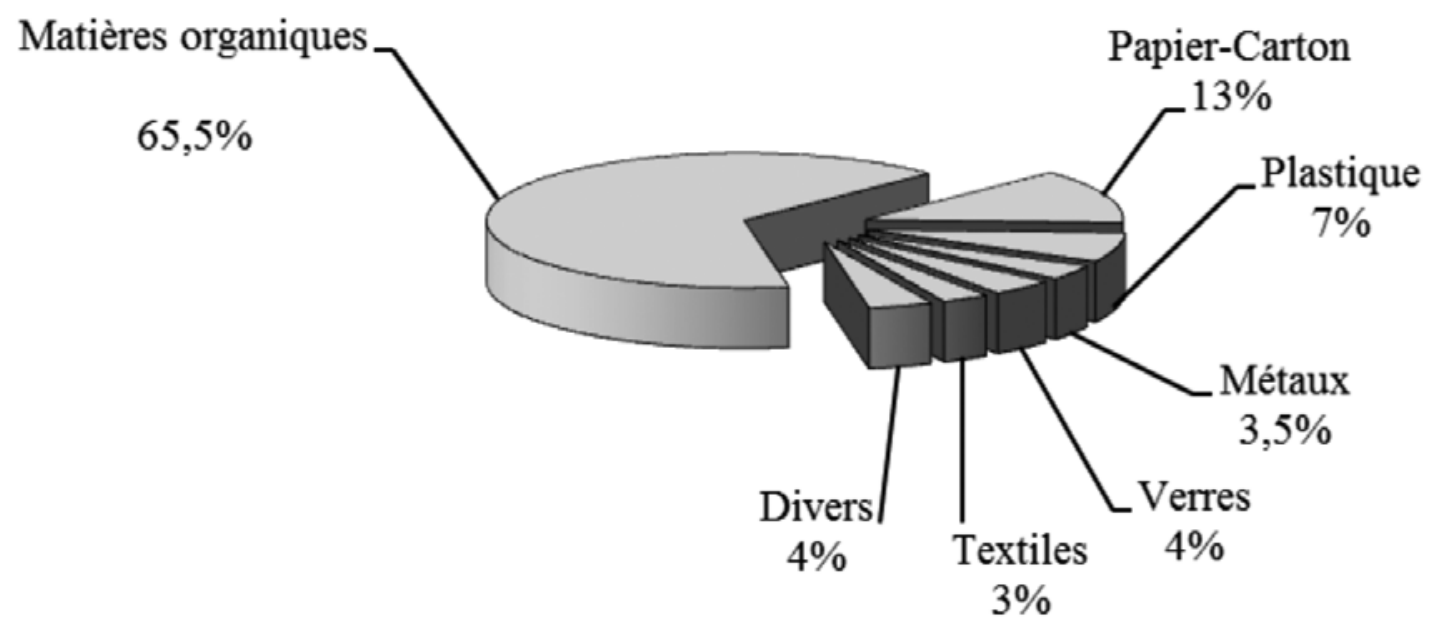

Figure 2. Composition moyenne des déchets ménagers de la ville de Mostaganem.

Tableau 2. Composition moyenne des ordures ménagères en fonction des saisons.

\begin{tabular}{l|c|c|c|c|}
\hline \multicolumn{1}{|c|}{ Catégorie } & Printemps & Eté & Automne & Hiver \\
\hline Humidité \% & 61,4 & 65,3 & 51,3 & 49,1 \\
\hline Matière organique & 65,6 & 80,3 & 72,8 & 64,8 \\
\hline Papier-Carton & 14,5 & 8,3 & 18,0 & 20,0 \\
Plastiques & 10,8 & 7,5 & 5,9 & 8,5 \\
Métaux & 1,5 & 1,0 & 1,0 & 2,2 \\
Verre & 2,9 & 1,3 & 0,9 & 2,8 \\
Textiles & 2,8 & 1,3 & 1,0 & 1,5
\end{tabular}

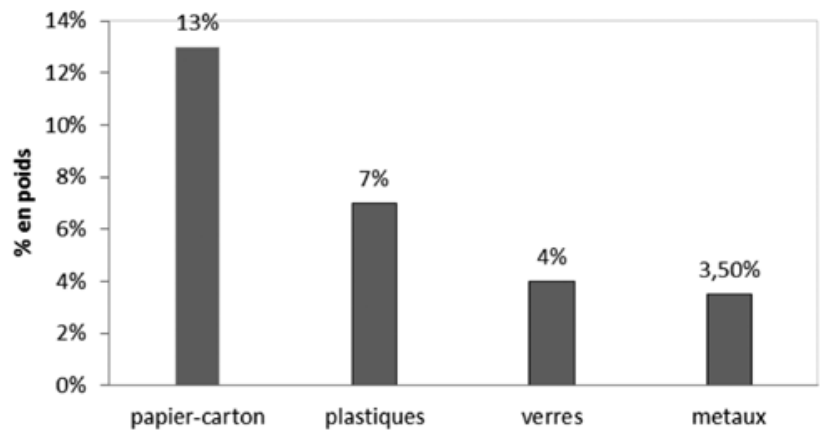

Figure 3.

Teneur en matériaux potentiellement recyclables.
Tandis que les déchets valorisables de France présentent une proportion très importante $(53,6 \%)$.

On peut dire qu'il est très important de s'intéresser et de se pencher vers la politique de gestion des résidus par la filière de récupération afin de réduire les quantités d'ordures déposées et de protéger ainsi l'environnement et servir l'économie.

\subsection{Discusion sur la filière de compostage}

Le compostage est un processus de fermentation par biodégradation aérobique des matières organiques carbonées et azotées en un produit appelé compost qui est hygiénique, de composition stable et riche en substances humiques. Les 
Tableau 3. Caractéristiques physico-chimiques des échantillons de déchets ménagers (valeurs moyennes).

\begin{tabular}{|c|c|c|c|c|c|c|c|c|}
\hline Paramètre & Chemouma & 5 juillet & Tigditt & C. ville & $\begin{array}{l}\text { Sala- } \\
\text { mandre }\end{array}$ & Kharouba & Moyenne & Norme \\
\hline Densité & 0,40 & 0,43 & 0,36 & 0,22 & 0,30 & 0,35 & 0,34 & - \\
\hline Humidité \% & 57 & 59 & 64 & 52 & 53 & 57 & 57 & - \\
\hline $\mathrm{pH}$ & 6,0 & 6,4 & 6,2 & 6,7 & 6,0 & 6,1 & 6,2 & $5,5-8$ \\
\hline $\mathrm{mS} / \mathrm{cm}$ & 2,7 & 1,2 & 3,1 & 3,8 & 1,8 & 2,0 & 2,4 & 3 \\
\hline Matière volatile \% & 61,6 & 61,8 & 61,6 & 62,0 & 64,0 & 62,2 & 62,2 & - \\
\hline Cendres \% & 38,4 & 38,2 & 38,4 & 38,0 & 36,0 & 37,8 & 37,8 & - \\
\hline Corganique \% & 27,8 & 29,0 & 27,1 & 25,0 & 29,0 & 28,0 & 27,6 & $5-30$ \\
\hline$N$ total $\%$ & 1,4 & 1,4 & 1,4 & 1,3 & $\mid, 4$ & 1,4 & 1,4 & $0,3-1,5$ \\
\hline $\mathrm{C} / \mathrm{N}$ & 19,9 & 20,7 & 19,4 & 19,2 & 20,7 & 20 & 20 & $20-35$ \\
\hline PCS (Kcal/Kg) & - & - & - & - & - & - & 1430 & \\
\hline
\end{tabular}

Tableau 4. Teneur en matériaux potentiellement recyclables dans différentes villes et pays.

\begin{tabular}{|c|c|c|c|c|c|c|c|}
\hline \multirow{4}{*}{$\begin{array}{c}\text { Taux de } \\
\text { récupération } \\
\%\end{array}$} & \multicolumn{4}{|c|}{ Algérie (MATE et PROGDEM, 2003) } & \multirow{3}{*}{$\begin{array}{c}\text { Jordanie } \\
\text { (Abu-Qudais, 2000) }\end{array}$} & \multirow{3}{*}{$\begin{array}{c}\text { Turquie } \\
\text { (Metin, 2003) }\end{array}$} & \multirow{3}{*}{$\begin{array}{c}\text { France } \\
(\text { Aina, 2006) }\end{array}$} \\
\hline & Mostaganem & Bejaia & Annaba & Tlemcen & & & \\
\hline & 27,5 & 26,8 & 28,6 & 26 & & & \\
\hline & \multicolumn{4}{|c|}{27,2} & 31,8 & 34 & 53,6 \\
\hline
\end{tabular}

conditions optimales pour un bon démarrage du compostage sont (CHARNAY, 2005 ; HAFID et al., 2002; HAUG, 1993) :

- taux d'humidité de 50 - 60\%

- rapport C/N de 20 - 35

- $\mathrm{pH}$ 5,5-8

- $\mathrm{O}_{2}$ minimum $5 \%$

Les déchets ménagers de la ville de Mostaganem se caractérisent par un taux d'humidité suffisant (57\%), un rapport C/N de l'ordre de l'ordre de 20, et un $\mathrm{pH}$ de 6,2. Ces valeurs sont favorables pour un traitement par le compostage.

\section{Conclusion}

Cette étude montre que la composition des déchets ménagers de la ville de Mostaganem dépend largement des conditions climatiques. L'analyse des résultats obtenus montre que les déchets ménagers de Mostaganem se répartissent en deux classes principales ;

- déchets recyclables (papier : 13\%, plastique : 7\%, verre : 4\%, métaux : 3,5\%) avec un taux de récupération total de 27,5\%, soit le 1/3 de la masse globale des déchets ménagers de la ville.
- déchets biodégradables représentés par la matière fermentescible qui constitue en moyenne $65,5 \%$ de la masse totale. Les ordures ménagères de la ville de Mostaganem renferment un taux d'humidité assez élevé de l'ordre de 57\% et une densité de 0,34. Le suivi du taux d'humidité en fonction du temps à montrer que ce dernier augmente durant la saison chaude $(80,3 \%)$ et diminue en période hivernale (64,8\%). Ces fluctuations reviennent essentiellement à la variété des aliments consommés selon les saisons. Les déchets ménagers de la ville de Mostaganem sont riches en matière organique et azotée et possède un taux d'humidité assez élevé. Ces résultats suggèrent que la filière de compostage serait bien adaptée comme mode de traitement de ces déchets de la ville de Mostaganem.

\section{Références bibliographiques}

ABU-QUDAIS M., ABU-QUDAIS H.A.; 2000. Energy content of municipal solid waste in Jordan and its potential utilization, Energy Conversion \& Management 4I, pp : 983-991.

AINA M.P., 2006, MSW Landfills Techniques in Developing Countries: Methodology and Experimental Applications. Doctorate Thesis. Limoges University France, $206 \mathrm{p}$.

ANNE, 1945, Dosage rapide du carbone organique des sols. Ann., Agr. 2., pp $161-172$. 
AUBERT G.; 1978, Méthodes d'analyses des sols, Marseille, Editions CRDP, $360 \mathrm{p}$.

BAYARD R., GOURDON R. 2007. Traitement biologique des déchets. Techniques de l'Ingénieur, J 3 966, pp : I-23.

CHARNAY F., 2005, Compostage des déchets urbains dans les Pays en développement : élaboration d'une

démarche méthodologique pour une production pérenne de compost. Thèse de doctorat. Université de Limoges, 277 p.

DOETSCH P., CHERIF M., LINS B., SCHNEIDER M. 2002, La station de compostage de la ville de Blida, Rapport de la coopération technique algeroallemand entre le Ministère de l'Aménagement du Territoire et de l'Environnement (MATE) et la Deutsche Gesellschaft für Technische Zusammenarbeit (GTZ) GmbH - Programme Gestion de l'Environnement, 63 p.

DOTREPPE J., GRISARON P., 1986, Déchets solides industriels et urbains Traitement, destruction et valorisation, Belgique, Ed. Cebedoc Liège, 84 p.

GILLET R.; 1985, Traité de gestion des déchets solides et son application aux pays en voie de développement, Vol I, Ed. OMS/ PUND, Copenhague, 397 p.

GILLET R., 1985, Traité de gestion des déchets solides et son application aux pays en voie de développement, Volo 2, PNUD, OMS, Copenhague, 537 p.

GIROD P., CREPEAU M., AFFHOLDER M.; 1982, Récupération et élimination des déchets, Textes officiels et jurisprudence, Paris, Editions Moniteur, 372 p

HAFID N., EL HADEK M., LGUIRATI A. et BOUAMRANE A. 2002. Evaluation d'une filière simplifiée de compostage des ordures ménagères. Déchets 25 pp : 13-17.

HAUG R.T., 1980, Compost Engineering, principes and practice, Michigan USA, Editions ANN ARBOR Science, 655 p

HAUG R.T. 1993, The practical handbook of compost engineering." Boca Raton, Florida.717 p

KHAN Z., ALI M., ABU-GHURRAH Z.H., I99I. New approaches for estimating energy content in MSV, ASCE journal of Environmental Engineering । 17(3), pp : 8-13.

MATE., 2003, Manuel d'information sur la gestion et l'élimination des déchets solides urbains, Alger, $162 \mathrm{p}$.

MATE., 2005, Rapport sur l'état et l'avenir de l'environnement 2005. 490 p.

MATE et PROGDEM,2003, Programme National pour la gestion des déchets solides en Algérie, Département d'état de l'Environnement, Alger, 2003.

MAYSTER L.Y. et DUFLONV., 1994, Déchets urbains, Nature et caractérisation, Suisse, Editions Presses Polytechniques et Universitaires Romandes, 219 p.

METIN E., EROZTUR K., NEYIM C., 2003. Solid waste management practices and review of recovery and recycling operations in Turkey, Waste Management 23, pp : 425-432

SCHUMACHER B.A., 2002, Methods for the determination of total organic carbon (TOC) in soils and sediments. Ecological Risk Assessment Support Center, Office of Research and Development. US Environmental Protection Agency. Las Vegas, NV. 25 p.

SOUMARE M.,TACK F.,VERLOO M.G.; 2003. Effects of municipal solid waste compost and mineral fertilization on plant growth in two tropical agricultural soils of Mali, Bioressource Technology 86, pp : I5-20.

TAD., 20 I0, Schéma directeur de gestion des déchets solides urbains de la ville de Mostaganem, Inspection de l'environnement, I 49 p.

ZOES V., DINEL H., PARE T., JAOUICH A.; 200I. Growth substrates mode from duck excreta enriched wood shavings and source-separated municipal solid waste compost and separates : physical and chemical characteristics, Bioressource Technology 78, pp : 21-30. 